\title{
Differentiable Programming for Image Processing and Deep Learning in Halide
}

\author{
TZU-MAO LI, MIT CSAIL \\ MICHAËL GHARBI, MIT CSAIL \\ ANDREW ADAMS, Facebook AI Research \\ FRÉDO DURAND, MIT CSAIL \\ JONATHAN RAGAN-KELLEY, UC Berkeley \& Google
}

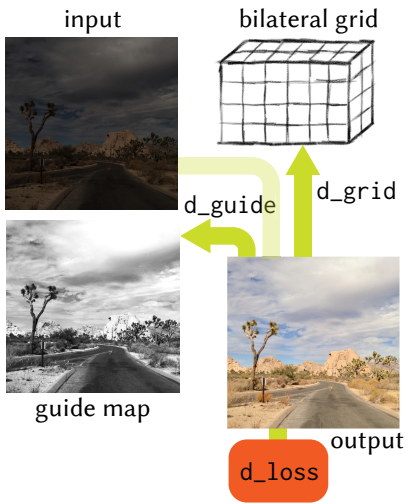

(a) Neural network operator: bilateral slicing

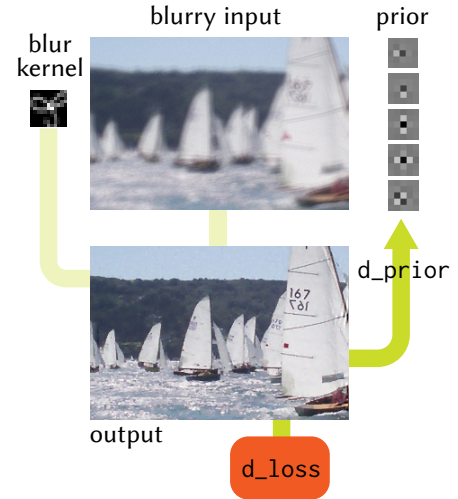

(b) optimizing the parameters of a forward image processing pipeline

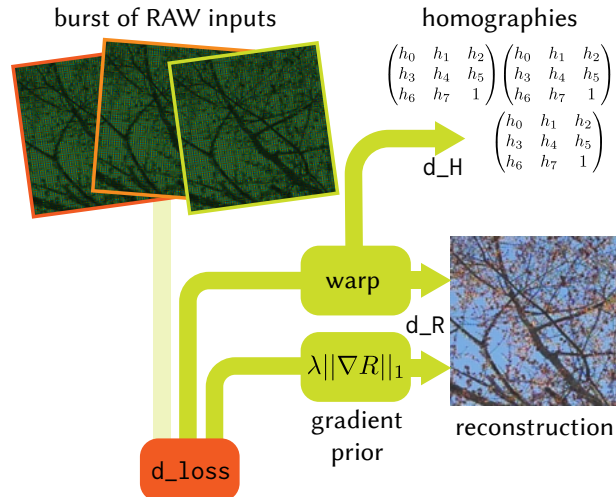

(c) optimizing the reconstruction and warping parameters of an inverse problem

Fig. 1. Our system automatically derives and optimizes gradient code for general image processing pipelines, and yields state-of-the-art performance on both CPUs and GPUs. This enables a variety of imaging applications, from training novel neural network layers (a), to optimizing the parameters of traditional image processing pipelines (b), to solving inverse reconstruction problems (c). To support these applications, we extend the Halide language with features to automatically and efficiently compute gradients of arbitrary programs. We also introduce a new automatic performance optimization that can handle the specific computation patterns of gradient computation. Using our system, a user can easily write high-level image processing algorithms, and then automatically derive high-performance gradient code for CPUs, GPUs, and other architectures. Images from left to right are from MIT5k dataset [Bychkovsky et al. 2011], ImageNet [Deng et al. 2009], and deep demosaicking dataset [Gharbi et al. 2016], respectively.

Gradient-based optimization has enabled dramatic advances in computational imaging through techniques like deep learning and nonlinear optimization. These methods require gradients not just of simple mathematical functions, but of general programs which encode complex transformations of images and graphical data. Unfortunately, practitioners have traditionally been limited to either hand-deriving gradients of complex computations, or composing programs from a limited set of coarse-grained operators in deep learning frameworks. At the same time, writing programs with the level of performance needed for imaging and deep learning is prohibitively difficult for most programmers.

We extend the image processing language Halide with general reversemode automatic differentiation (AD), and the ability to automatically optimize the implementation of gradient computations. This enables automatic

Authors' addresses: Tzu-Mao Li, MIT CSAIL, tzumao@mit.edu; Michaël Gharbi, MIT CSAIL, gharbi@mit.edu; Andrew Adams, Facebook AI Research, andrew.b.adams@ gmail.com; Frédo Durand, MIT CSAIL, fredo@mit.edu; Jonathan Ragan-Kelley, UC Berkeley \& Google, jrk@berkeley.edu.

Permission to make digital or hard copies of part or all of this work for personal or classroom use is granted without fee provided that copies are not made or distributed for profit or commercial advantage and that copies bear this notice and the full citation on the first page. Copyrights for third-party components of this work must be honored. For all other uses, contact the owner/author(s).

(c) 2018 Copyright held by the owner/author(s).

0730-0301/2018/8-ART139

https://doi.org/10.1145/3197517.3201383 computation of the gradients of arbitrary Halide programs, at high performance, with little programmer effort. A key challenge is to structure the gradient code to retain parallelism. We define a simple algorithm to automatically schedule these pipelines, and show how Halide's existing scheduling primitives can express and extend the key $\mathrm{AD}$ optimization of "checkpointing."

Using this new tool, we show how to easily define new neural network layers which automatically compile to high-performance GPU implementations, and how to solve nonlinear inverse problems from computational imaging. Finally, we show how differentiable programming enables dramatically improving the quality of even traditional, feed-forward image processing algorithms, blurring the distinction between classical and deep methods.

CCS Concepts: • Computing methodologies $\rightarrow$ Graphics systems and interfaces; Machine learning; Image processing;

Additional Key Words and Phrases: image processing, deep learning, automatic differentiation

\section{ACM Reference Format:}

Tzu-Mao Li, Michaël Gharbi, Andrew Adams, Frédo Durand, and Jonathan Ragan-Kelley. 2018. Differentiable Programming for Image Processing and Deep Learning in Halide. ACM Trans. Graph. 37, 4, Article 139 (August 2018), 13 pages. https://doi.org/10.1145/3197517.3201383 


\section{INTRODUCTION}

Optimization and end-to-end learning are driving rapid progress in graphics and imaging, by viewing either the output image or large sets of pipeline parameters as unknowns, e.g. [Barron and Poole 2016; Gharbi et al. 2017; Heide et al. 2014; Jaderberg et al. 2015]. Key to this progress is the surprising power of gradient-based optimization methods to find solutions to nonlinear objectives over large sets of unknowns. Unfortunately, the computation of gradients remains a challenge in the general case, especially when performance is paramount such as for training neural networks or when solving for images via optimization. Practitioners have to either manually derive gradients or they are limited to the composition of building blocks offered by deep learning libraries. The result is often inefficient, and when users decide to stray from existing operators, the implementation of fast GPU derivative code is a major undertaking.

At first glance, modern machine learning frameworks like PyTorch, TensorFlow or CNTK [Abadi et al. 2015; Paszke et al. 2017; Yu et al. 2014] seem like appealing environments for new gradientbased graphics algorithms. When limited to their walled-gardens of pre-made, coarse-grained operations, these frameworks provide high-performance kernel implementations and automatic differentiation (AD) through chains of operations. As general programming languages, however, they are a poor fit for many imaging applications. Building new algorithms requires contorting a problem into complex and tangled compositions of existing building blocks. Even when done successfully, the resulting implementation is often both slow and memory-inefficient, saving and reloading entire arrays of intermediate results between each step, causing costly cache misses.
Consider the following example. A recent neural network-based image processing approximation algorithm was built around a new "bilateral slicing" layer based on the bilateral grid [Chen et al. 2007; Gharbi et al. 2017]. At the time it was published, neither PyTorch nor TensorFlow was even capable of practically expressing this computation. ${ }^{1}$ As a result, the authors had to define an entirely new operator, written by hand in about 100 lines of CUDA for the forward pass and 200 lines more for its manually-derived gradient (Fig. 2, right). This was a sizeable programming task which took significant time and expertise. While new operations-added in just the last six months before the submission of this paper-now make it possible to implement this operation in 42 lines of PyTorch, this yields less than $1 / 3$ rd the performance on small inputs and runs out of memory on realistically-sized images (Fig. 2, middle). The challenge of efficiently deriving and computing gradients for custom nodes remains a serious obstacle to deep learning.

This pattern is ubiquitous. New custom nodes require major effort to implement correctly and efficiently, making it hard to experiment. Similarly, general image processing pipelines often do not map well to deep learning toolboxes. As a result, most researchers limit themselves to consider only operations which are already well-supported by existing frameworks, while NVIDIA and the framework developers must constantly expand the set of native operations. (There are currently at least 12 different kinds of convolution operator, alone, in TensorFlow.) The only alternative is to invest orders of magnitude

\footnotetext{
${ }^{1}$ Technically, TensorFlow graphs are Turing-complete, thanks to their inclusion of a while loop node. However, implementing the algorithm at this level would be both incredibly complex and run at least thousands of times slower.
}

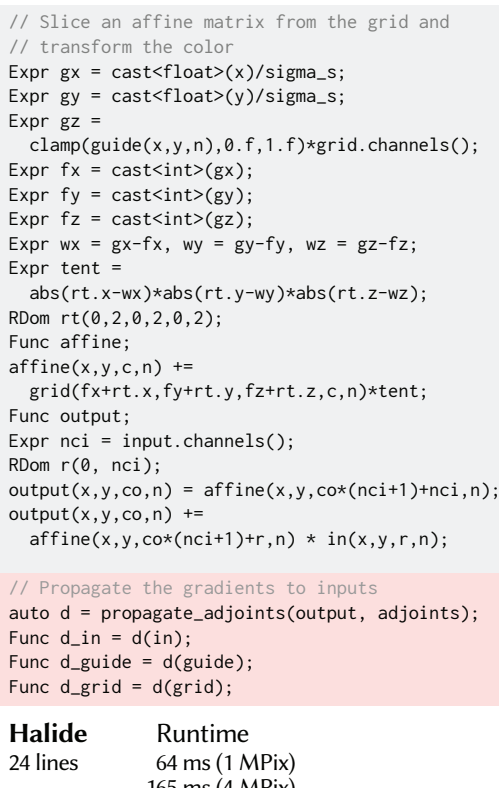

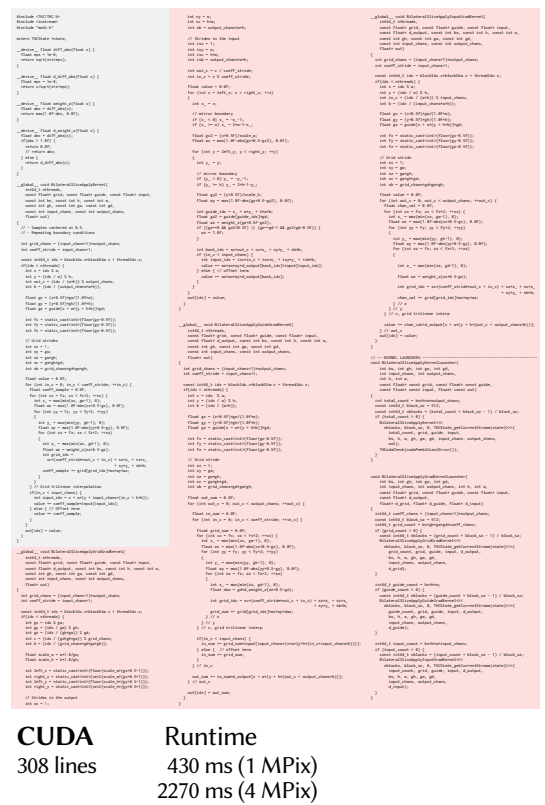

Fig. 2. Implementations of the forward and gradient computations of the bilateral slicing layer [Gharbi et al. 2017] in Halide, PyTorch, and CUDA. Using our automatic differentiation and scheduling extensions, the Halide implementation is clear, concise, and fast. The PyTorch implementation is modestly more complex, but runs $20 \times$ slower on a $1 k \times 1 k$ input, fails to complete (out of memory on a 12 GB NVIDIA Titan Xp) on a $2 k \times 2 k$ input, and is only possible thanks to new operators added to PyTorch since the original publication. The CUDA implementation, developed by the original authors, is not only complex (an order of magnitude larger than either Halide or PyTorch), but is dominated by hand-derived gradient computations. It is faster than PyTorch and scales to larger inputs, but is still about $10 \times$ slower than the Halide version. Note: code size includes a few lines beyond the core logic shown for both Halide and PyTorch. 
more effort in developing custom operations, hand-deriving, reimplementing, and debugging gradient code for every change during the development of a new algorithm.

Recently, the Halide domain-specific language [Ragan-Kelley et al. 2012, 2013] has enabled the implementation of high-performance image-processing pipelines. It is an effective solution to implementing custom nodes and general image processing pipelines, but it still requires the manual derivation of gradients. Furthermore, our experience shows that the computation pattern of derivatives differs from that of forward code, which causes existing automatic performance optimizations in Halide to fail. Critically, the current built-in Halide autoscheduler does not support GPU schedules.

In this paper, we extend Halide with methods to automatically and efficiently compute the gradients of arbitrary Halide programs using reverse-mode automatic differentiation (Sec. 4). This transformation supports all existing features in the language.

Building atop Halide has several advantages. It provides a concise, natural language in which to express image processing computations, and for which there is already a library of existing algorithms. The Halide compiler portably targets numerous processor and accelerator architectures, from mobile CPUs, to image processing DSPs, to data center GPUs, and supports compilation to very highperformance code. Finally, Halide's existing language and scheduling constructs compose with reverse-mode $\mathrm{AD}$ to naturally express and generalize essential optimizations from the traditional $\mathrm{AD}$ literature (Sec. 4.3). Key to making our compiler transformation work are a scatter-to-gather conversion algorithm which preserves parallelism (Sec. 4.2.1), and a simple automatic scheduling algorithm specialized to the patterns that appear in generated gradient code (Sec. 4.4). Halide's existing system of powerful dependence analyses is essential for both. In contrast to traditional Halide, automatic scheduling is critical given the complexity of the automaticallygenerated gradient code.

Using our new automatic gradient computation and automatic scheduler, we show how we can easily implement three recentlyproposed neural network layers using code that is both faster and significantly simpler than the authors' original custom nodes written in $\mathrm{C}++$ and CUDA (Sec. 5.1). For example, the aforementioned bilateral slicing layer is expressed in 24 lines of Halide (Fig. 2, left), including just four lines to compute and extract its gradients, while compiling automatically to an implementation about $10 \times$ faster than the authors' original handwritten CUDA, and $20 \times$ faster than a more limited version in PyTorch. We believe that this ease of implementation and performance tuning will dramatically facilitate prototyping, by delivering both automatic gradients and high performance at the outset of experimentation, not after-the-fact once the usefulness of a node has been established, but as soon as experimentation begins.

We also argue that this approach of gradient-based optimization through arbitrary programs is useful outside the traditional deep learning applications which have popularized it. Our vision is that any image-processing pipelines can benefit from an automatic tuning of internal parameters. Currently, this step is usually done by hand through user trial-and-error. The availability of automatic derivatives makes it possible to systematically optimize any internal parameter of an image processing pipeline, given some output objectives. This is especially appealing when gradients are available in the same language used for high-performance code deployment. We show how to significantly improve the performance of two traditional image processing algorithms by automatically optimizing their key parameters and filters (Sec. 5.2). We also implement a novel joint burst demosaicking and superresolution algorithm by inverting a forward image formation model including warps by unknown homographies, solving for the image and homographies simultaneously (Sec. 5.3). Finally, we show the versatility of our approach and implement a lens design optimization by differentiating an optical simulator (Sec. 5.4).

\section{RELATED WORK}

\subsection{Automatic differentiation}

Automatic differentiation is a collection of techniques to numerically evaluate the derivatives of a computer program [Griewank and Walther 2008]. Automatic differentiation is distinct from both finite differences and symbolic differentiation. It exploits the structure of the computation graph by recursively applying the chain rule, and it synthesizes a new program that computes the derivatives, instead of closed-form algebraic expressions. To compute the gradient of a scalar output, traversing the computation graph backwards from the output to propagate the adjoints to all the inputs gives the same time complexity as the original program (e.g. [Linnainmaa 1970; Werbos 1982]. Automatic differentiation has been rediscovered as "backpropagation" for neural networks [Rumelhart et al. 1986]).

Although the time complexity of the gradient computation matches that of the original program, the backward traversal can use significantly more memory than the forward pass. Traditional automatic differentiation systems trade off between memory and run time using a checkpointing strategy [Volin and Ostrovskii 1985]. Our system allows the user to explore the space of trade-offs using scheduling mechanisms provided by the Halide language (Sec. 4.3).

Many automatic differentiation frameworks have been developed for general programming languages [Bischof et al. 1992; Griewank et al. 1996; Hascoet and Pascual 2013; Hogan 2014; Wiltschko et al. 2017], but general programming languages can be cumbersome for image processing applications. Writing efficient image processing code requires enormous efforts to take parallelism, locality, and memory consumption/bandwidth into account [Ragan-Kelley et al. 2012]. These difficulties are compounded when we also want to compute derivatives. Other recent packages provide higher level, highly optimized differentiable building blocks for users to assemble their program [Abadi et al. 2015; Bergstra et al. 2010; Paszke et al. 2017; Yu et al. 2014]. These packages are efficient when the algorithm to be implemented can be conveniently expressed by combining these building blocks. But it is quite common for users to write their own custom operators in low-level $\mathrm{C}++$ or CUDA to extend a package's functionalities. This means that users have to write code for both the forward program and its gradients, and make sure they are correct, consistent and reasonably efficient. This can be tedious, error-prone and challenging to maintain. Using our approach, one can simply write the forward program. Our algorithm generates the derivatives and, thanks to Halide's decoupling of algorithm and schedule and our automatic scheduler, provides convenient handles to easily produce efficient code. 


\subsection{Image processing languages}

Our work builds on the Halide [Ragan-Kelley et al. 2012] image processing language, which we briefly introduce in Sec. 3.

The Opt language [Devito et al. 2017] focuses on nonlinear least squares problems. It provides language constructs to describe least squares cost and automatically generates solvers. It uses the $\mathrm{D}^{*}$ algorithm [Guenter 2007] to generate derivatives. The ProxImaL [Heide et al. 2016] language, on the other hand, focuses on solving inverse problems using proximal gradient algorithms. The language provides a set of functions and their corresponding proximal operators. It then generates Halide code for optimization. Our system can be used to generate the adjoints required by new ProxImaL operators. These languages focus on a specific set of solvers, namely nonlinear least squares and proximal methods, and provide high-level interfaces to them. On the other hand, we deal with any problem that requires the gradient of a program. Our system can also be used to solve for unknowns other than images, such as optimizing the hyperparameters of an algorithm or jointly optimizing images and parameters. Sec. 5.3 demonstrates this with some examples.

Recently, there have been attempts to automatically speed-up image processing pipelines [Mullapudi et al. 2016, 2015; Yang et al. 2016]. We developed a new automatic scheduler in Halide with specialized mechanisms for parallel reductions [Suriana et al. 2017], which often occur in the derivatives of image processing code. Our system could further benefit from future developments in automatic code optimization.

\subsection{Learning and optimizing with images}

Gradient-based optimization is commonly used in image processing. It has been used for image restoration [Rudin et al. 1992], image registration [Zitova and Flusser 2003], optical flow estimation [Horn and Schunck 1981], stereo vision [Barron and Poole 2016], learning image priors [Roth and Black 2005; Ulyanov et al. 2017] and solving complex inverse problems [Heide et al. 2014]. Our work alleviates the need to manually derive the gradient in such applications, which enables faster experimentation. Deep learning has revitalized an interest in building differentiable forward image processing pipelines whose parameters can be tuned by stochastic gradient descent. Successful instances include image restoration [Gharbi et al. 2016; Zhang et al. 2017], photographic enhancement [Xu et al. 2015], and applications such as colorization [Iizuka et al. 2016; Zhang et al. 2016], and style transfer [Gatys et al. 2016; Luan et al. 2017]. Some of these methods call for custom operators [Gharbi et al. 2017; Ilg et al. 2017; Jaderberg et al. 2015], typically not available in mainstream frameworks. For these custom operators, the forward and gradient operations are implemented manually. Our work provides a convenient way to explore new custom computations.

\section{THE HALIDE PROGRAMMING LANGUAGE}

Our system extends the Halide programming language. We will give a brief overview of the constructs in Halide that are relevant to our system. For more detail on Halide, see the original papers [RaganKelley et al. 2012, 2013] and documentation. ${ }^{1}$

\footnotetext{
${ }^{1}$ http://halide-lang.org/
}

Halide is a language designed to make it easy to write highperformance image- and array-processing code. The key idea in Halide is the separation of a program into the algorithm, which specifies what is computed, and the schedule, which dictates the order of computation and storage. The algorithm is expressed as a pure functional, feed-forward pipeline of arithmetic operations on multidimensional grids. The schedule addresses concerns such as tiling, vectorization, parallelization, mapping to a GPU, etc. The language guarantees that the output of a program depends only on the algorithm and not on the schedule. This frees the user from worrying about low-level optimizations while writing the high-level algorithm. They can then explore optimization strategies without unintentionally altering the output.

By adding automatic differentiation to Halide, we build on this philosophy. To create a differentiable pipeline, the user no longer needs to worry about the correctness and efficiency of the gradient code. With the sole specification of a forward algorithm, our system synthesizes the gradient algorithm. Optimization strategies can then be explored for both, either manually or with an auto-scheduler.

The following code shows an example Halide program that performs gamma correction on an image and computes the $L^{2}$ norm between the output and a target image:

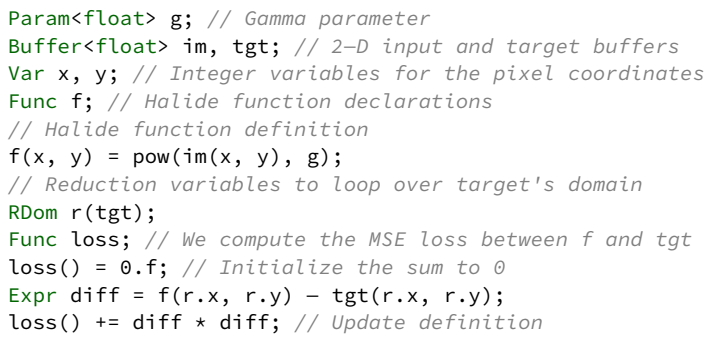

Halide is embedded in $\mathrm{C}++$. Halide pipeline stages are called functions and represented in code by the $\mathrm{C}++$ class Func. Each Halide function is defined over an $\mathrm{n}$-dimensional grid. The definition of a function comprises:

- an initial value that specifies a value for each grid point.

- optional recursive updates that modify these values in-place.

The function definitions are specified as Halide expressions (objects of type Expr). Halide expressions are side-effect-free, including arithmetic, logical expressions, conditionals, and calls to other Halide functions, input buffers, or external code (such as sin or exp).

Reduction operators, such as summation or general convolution, are implemented through recursive updates of a Halide function. The domain of a reduction is represented in code as an RDom, which implies a loop over that domain. All loops in Halide are implicit, whether over the domain of a function or a reduction.

Scheduling is expressed through methods exposed on Func. There are many scheduling operators, which transform the computation to trade off between memory bandwidth, parallelism, and redundant computation. Halide lowers the schedule and algorithm into a set of loop nests and kernels. These are then compiled to machine code for various architectures. We use the CUDA and x86 backends for the applications demonstrated in this paper. 


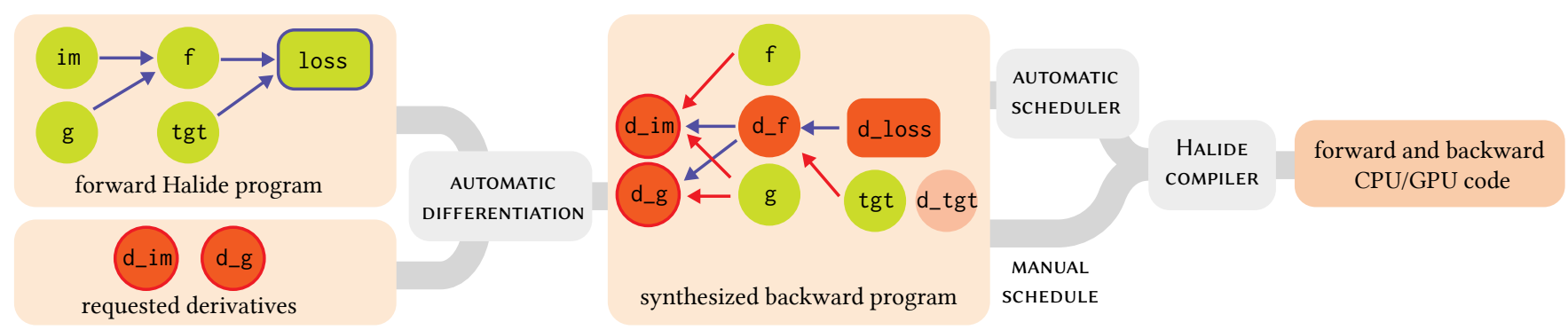

Fig. 3. Overview of our compiler. The user writes a forward Halide program as they would normally. Then, they specify the set of outputs and gradients the system should produce. Our automatic differentiation generates new Halide functions that implement the requested gradients. The user can either manually schedule the pipeline or use our automatic scheduler. Finally, the Halide compiler generates machine code for the scheduled forward and backward algorithms.

\section{METHOD}

To use our system, a programmer first writes a forward Halide algorithm. They then may request the derivative of some scalar loss with respect to any Halide function, image buffer, or parameter in the pipeline. Our automatic differentiation system visits the graph of functions that describes the forward algorithm and synthesizes new Halide functions that implement the gradient computation (Sec. 4.1). The programmer can either specify the schedule for these new functions manually or use our automatic scheduler (Sec. 4.4). Unlike Halide's built-in auto-scheduler [Mullapudi et al. 2016], ours recognizes patterns that arise when reversing the computation graph (Sec. 4.2.1). Figure 3 illustrates this workflow.

\subsection{High-level strategy}

We assume we wish to compute the derivatives of some scalar $\mathcal{L}$, typically a cost function to be minimized. Our system implements reverse-mode automatic differentiation, which computes the gradient with the same time complexity as the forward function (e.g. [Griewank and Walther 2008]). We propagate the adjoints $\frac{\partial \mathcal{L}}{\partial g}$ to each function in the forward pipeline $g$, until we reach the inputs. The adjoints of the inputs are the components of the gradient.

Specifically, given a Halide program represented as a graph of Halide functions, we traverse the graph backwards from the output and accumulate contributions to the adjoints using the chain rule. Halide function definitions are represented as expression trees, so within each function we perform a similar backpropagation through the expression tree, propagating adjoints to all leaves.

A key difference between our algorithm and traditional automatic differentiation arises when an expression is a Halide function call. We need to construct a computation which accumulates adjoints onto the called function in the face of non-trivial data dependencies between the two functions. Sec. 4.2 describes this in detail.

We illustrate our algorithm on the simple example in Sec. 3, which performs gamma correction on an image and computes the $L^{2}$ distance between the output and some target image. To compute the gradients of the $L^{2}$ distance with respect to the input image and the gamma parameter, one would write:

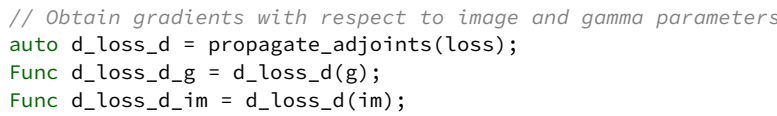

Throughout the paper, we use the convention that prefixing a function's name with d_ refers to the gradient of that Halide function. We added a key language extension, propagate_adjoints, to Halide. It takes a scalar Halide function and generates gradients in the form of new Halide functions for every Halide function, buffer, and real number parameter the output depends on. Our system can also be used as a component in other automatic differentiation systems that compute gradients. In this case the user can specify a nonscalar Halide function and a buffer representing the adjoints of the function. Figure 3 shows the computational graph for both the forward and backward (gradient) computations.

\subsection{Differentiating Halide function calls}

A key difference between automatic differentiation in Halide and traditional automatic differentiation is that Halide functions are defined on multi-dimensional grids, so function calls and the elements on the grids can have non-trivial aggregate interactions.

Given each input-output pair of Halide functions, we synthesize a new Halide function definition that accumulates the adjoint of the output function onto the adjoint of the input. For performance, we want these new definitions to be as parallelizable as possible.

4.2.1 Scatter-gather conversion. Two cases require special care for correctness and efficiency. The first and most important case occurs when each output element reads and combines multiple input values. This happens for example in the simple convolution of Figure 4(a). We call this pattern a gather operation.

When computing gradients in reverse automatic differentiation, the natural reverse of this gather is a scatter operation: each input writes to multiple elements of the output. Scattering operations, however, are not naturally parallelizable since they may lead to race conditions on write. For this reason, we want to convert scatters back to gathers whenever possible. We do this by shearing the iteration domain (e.g. [Lamport 1975]). To illustrate this transformation, consider the following code that convolves a 1D signal with a kernel, also illustrated in Figure 4(a):

Func output;

$\operatorname{output}(x)=\operatorname{input}(x-r \cdot x) \star \operatorname{kernel}(r \cdot x)$;

Assume that we are interested in propagating the gradient to input. This is achieved by reversing the dependency graph between the input and output variables as shown in Figure 4(b). In code, this transformation would yield:

RDom ro;

d_input (ro.y - ro.x $)+=$ d_output $($ ro.y $) * \operatorname{kernel}($ ro. $x)$; 
where ro. $x$ iterates over the original $r . x$, and ro.y iterates over the domain of output. For each argument in the calls to input, we replace the pure variables ( $x$ here) with reduction variables that iterate over the domain of the output (in this case ro.y). $r . x$ is renamed to ro. $x$ so we can merge the reduction variables into a single reduction domain ro.

This new update definition cannot be computed in parallel over ro. y since multiple ro.y - ro.x may write to the same memory location. A more efficient way to compute the update, illustrated in Figure 4(c), is to rewrite the same computation as follows:

d_output $(x)=\operatorname{select}\left(x>=a \& \& x<b, d \_o u t p u t(x), 0 . f\right)$;

$d_{-}$input $(x)+=d_{-}$output $(x+r \cdot x) \star \operatorname{kernel}(r \cdot x)$;

where $a$ and $b$ are the bounds of output. By shearing the iteration domain with the variable substitution $x=$ ro. $y-$ ro. $x$, we have made $d_{-}$input parallelizable over $x$. Because Halide only iterates over rectangles, and the sheared iteration domain is no longer a rectangle, we add a zero-padding boundary condition to d_output, and iterate over a conservative bounding box of the sheared domain:
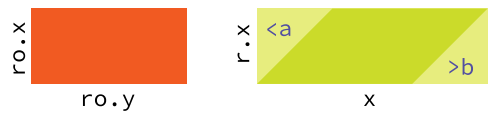

We use Halide's equation-solving tools to deduce the variable substitution to apply. For each argument in a function call, we construct an equation e.g. $u=x-r_{x}$ and solve for $x$. Importantly, we solve for the smallest interval of $x$ where the condition holds, since $x$ may map to multiple values. This may introduce new reduction variables, as in the following upsampling operation:

$\operatorname{output}(x)=\operatorname{input}(x / 4)$;

Since $x$ is an integer, 4 values in input are used to produce each value of output. Accordingly, our converter will generate the following adjoint code:

RDom $r(\odot, 4)$; // loops from $\odot$ to 3

d_output $(x)=d_{-}$input $(4 \star x+r \cdot x)$

If any step of this procedure fails to find a solution, we fall back to a general scattering operation. It is still possible to parallelize general scatters using atomics. We added atomic operations to Halide's GPU backend to handle this case. A general scatter with atomics usually remains significantly less efficient than our transformed code. For instance, the backward pass of a 2D convolution layer applied to a $16 \times 16 \times 256 \times 256$ input takes $68 \mathrm{~ms}$ using atomics and $6 \mathrm{~ms}$ with our scatter-to-gather conversion.

Listing 1 shows some derivatives our system would generate for the bilateral slicing example in the left of Figure 2.

4.2.2 Handling partial updates. The second case which requires special care arises when reversing partial updates to a function. For example, consider the following forward code:

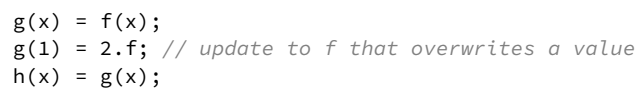

When backpropagating the adjoints, we need to propagate correctly through the chain of update definitions. While $h(x)$ depends on $f(x)$ for most $x(\operatorname{via} g(x))$, this is not true for $x==1$. The update definition to $g$ hides the previous dependency on $f(1)$. The corresponding gradient code is:

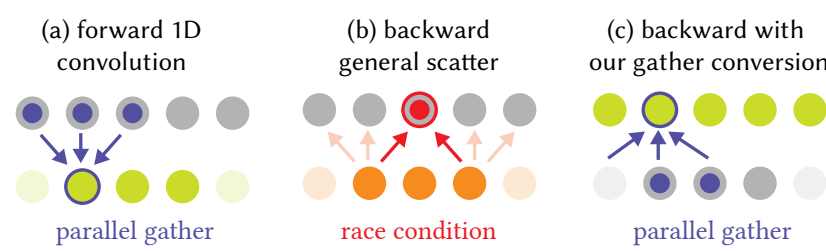

Fig. 4. Our scatter-to-gather conversion enables efficient, parallel code. In this example of a 1D 3-tap convolution, each dot represents a value in the input (resp. output) array. The forward computation (a) produces an output value from three inputs (the faded dots account for boundary conditions). This 3-tap reduction can easily be run in parallel over the output buffer (green dots). Computing the adjoint operator by simply reversing the dependency graph (b), that is by looping in parallel over the output nodes (orange), leads to race conditions since two inputs might need to write to the same location in the input's adjoint buffer (highlighted in red). This is a common issue with general scattering operations. Using our scatter-to-gather conversion, we convert this backward operation to a reduction over d_out (the adjoint of a convolution is a correlation). In turn, this transformed computation is readily parallelized over d_out's domain (c).

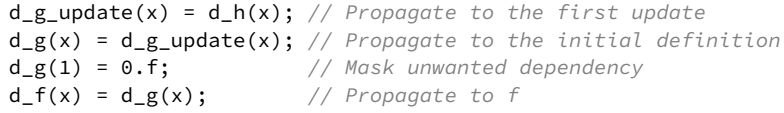

In general, if we detect different update arguments between two consecutive function updates (in the example above, $g(1)$ is different from $g(x)$ ), we mask the adjoint of the first update to zero using the update argument of the second update.

\subsection{Checkpointing}

Reverse-mode automatic differentiation on complex pipelines must traditionally deal with a difficult trade-off. Memoizing values from the forward evaluation to be reused in the reverse pass saves compute, but costs memory. Even with unlimited memory, bandwidth is limited, so it can be more efficient to recompute values. In automatic differentiation systems this trade-off is addressed with checkpointing [Volin and Ostrovskii 1985], which reduces memory usage by recomputing parts of the forward expressions. However, this is just a specific instance of the general recomputation-vs-memory trade-off already addressed by Halide's scheduling primitives.

For each function, we can decide whether to create an intermediate buffer for later reuse (the compute_root () construct), or recompute values at every call site (the compute_inline() construct). We can also compute these values at some intermediate granularity, i.e., by setting its computation somewhere in the loop nest of their consumers (the compute_at () construct). Halide also allows checkpointing across different Halide pipelines by using a global cache (the memoize() construct). This is useful when the forward pass and backward pass are in separately-compiled units.

As an example, consider the following 2D convolution implementation in Halide:

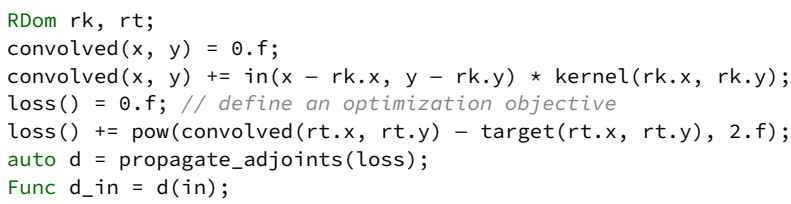




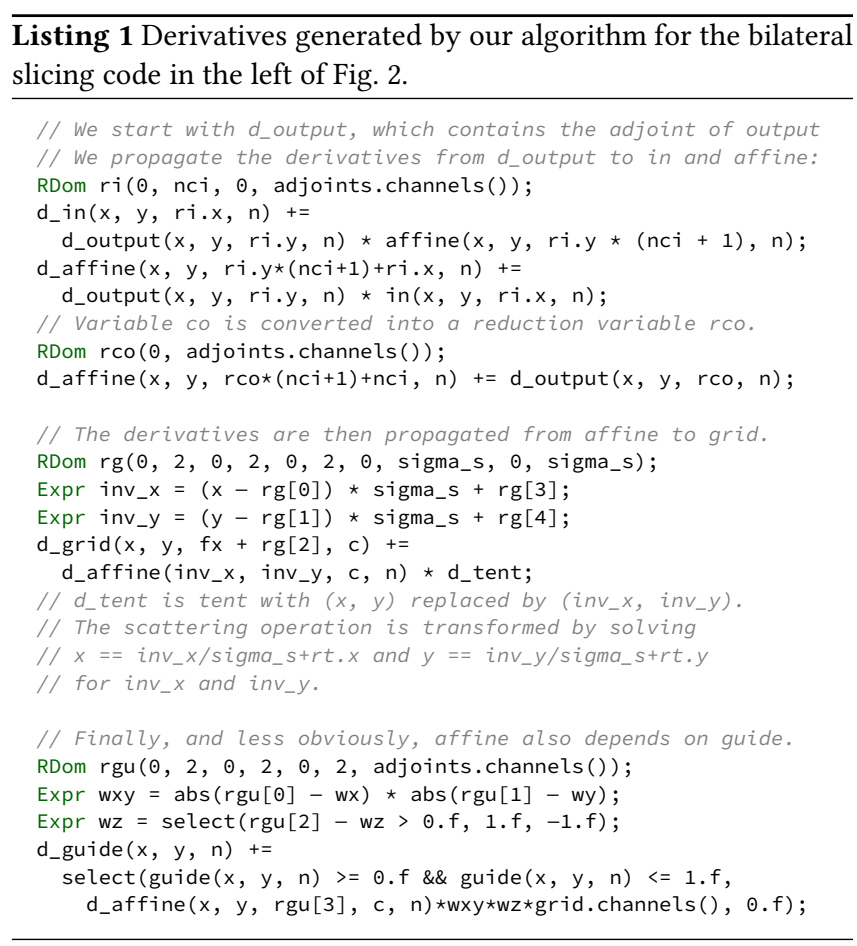

We are interested in d_in, the gradient of loss with respect to in. It is given by a correlation of $2 *$ (convolved-target) with kernel, which depends on the values of convolved. Using the scheduling handles provided by Halide, we can easily decide whether to cache the values of convolved for the gradient computation. For example, if we write:

convolved.compute_root();

the values of convolved are computed once and will be fetched from memory when we need them for the derivative $d_{-} i n$. On the other hand, if we write:

convolved.compute_inline();

the values of convolved are computed on-the-fly and no buffer is allocated to store them. This can be advantageous when the convolution kernel is small (say $2 \times 1$ ) since this preserves memory locality, or when the pipeline is much longer and we cannot afford to store every intermediate buffer.

Halide provides scheduling primitives that are more general than binary checkpointing decisions. Fine-grained control over the schedule allows exploration of memory/recomputation trade-offs in the forward and gradient code. For instance, we can interleave the computation and storage of convolved with the computation of another Halide function that consumes its value (in this case $d_{-} i n$ ). The following code instructs Halide to compute and store a tile of convolved for each $32 \times 32$ tile of $d_{-}$in computed. This offers a potentially faster balance between computing all of convolved before backpropagation, or recomputing each of its pixels on-demand:

d_in. $\operatorname{compute} \_r o o t() \cdot \operatorname{tile}(x, y, x i, y i, 32,32)$;

convolved.compute_at(d_in, $x)$; // compute at each tile of $d_{-}$in

We timed the three schedules above by computing d_in. With multi-threading and vectorization on a CPU, on an image with size of $2560 \times 1600$ and kernel size $1 \times 5$, the compute_inline schedule takes 5.6 milliseconds while the compute_root schedule takes 10.1 milliseconds and the compute_at schedule takes 9.7 milliseconds. On the same image but with kernel size $3 \times 5$, the compute_inline schedule takes 66.2 milliseconds while the compute_root schedule takes 18.7 milliseconds and the compute_at schedule takes 12.3 milliseconds.

\subsection{Automatic scheduling}

Halide's built-in auto-scheduler [Mullapudi et al. 2016] navigates performance trade-offs well for stencil pipelines, but struggles with patterns that arise when reversing their computational graph (Sec. 4.2.1). In particular, it does not try to optimize large reductions, like those needed to compute a scalar loss. It also does not generate GPU schedules. We therefore implemented a custom automatic scheduler for gradient pipelines.

Similar to Halide's built-in auto-scheduler, we ask the user to provide an estimate of the input and output buffer sizes. We then infer the extent of all the intermediate functions' domains.

Our automatic scheduler checkpoints (compute_root) any stage that scatters or reduces, along with those called by more than one other function. We leave any other functions to be recomputed ondemand (compute_inline). For the checkpointed functions, we tile the function domain and parallelize the computation over tiles when possible. Specifically, on CPUs, we split the function's domain into 2 D tiles $(16 \times 16)$ and launch CPU threads for each tile, vectorizing the innermost dimension inside a tile. On GPUs, we split the domain into 3D tiles $(16 \times 16 \times 4)$. The tiles are mapped to GPU blocks, and elements within a tile to GPU threads. In both cases, we tile the first two (resp. three) dimensions of the function's domain that are large enough. We split the domain if its dimensionality is too low.

If the function's domain is not large enough for tiling, and the function performs a large associative reduction, we transform it into a parallel reduction using Halide's rfactor scheduling primitive [Suriana et al. 2017]. This allows us to factorize the reduction into a set of partial reductions which we compute in parallel and a final, serial reduction. Like before, we find the first two dimensions of the reduction domain which are large enough for tiling. We reduce the tiles in parallel over CPU threads (resp. GPU blocks). Within each 2D tile, we vectorize (resp. parallelize over GPU threads) the column-wise reductions. We also implemented a multi-level parallel reduction schedule but found it unnecessary in the applications presented. When compiling to GPUs, if both the function domain and the reduction domain are large enough for tiling, but the recursive update does not contain enough pure variables for parallelism, we parallelize the reduction using atomics.

To allow for control over checkpointing, the automatic scheduler decisions can be overridden. We ask the user to provide optional lists of Halide functions they do or do not want to inline. We currently do not use compute_at in our automatic scheduler.

\section{APPLICATIONS \& RESULTS}

We generate gradients for pipelines in three groups of applications. First, we show that our system can be integrated into existing deep learning systems to more easily develop new, custom operators. Second, we show that we can improve existing image processing pipelines by optimizing their internal parameters on a dataset of training images. Finally, we show how to use our derivatives to solve inverse imaging problems (i.e., optimizing for the image itself). 
Unless otherwise specified, we use our automatic scheduler (Sec. 4.4) to schedule all the applications throughout the section (i.e., for both the forward code and the derivatives we generate). Therefore, our implementation only requires the programmer to specify the forward pass of the algorithm.

\subsection{Custom neural network layers}

The class of computations expressible with deep learning libraries such as Caffe [Jia et al. 2014], PyTorch [Paszke et al. 2017], TensorFlow [Abadi et al. 2015], or CNTK [Yu et al. 2014] is growing increasingly rich. Nonetheless, it is still common for a practitioner to require a new, custom node tailored to their problem. For instance, TensorFlow offers a bilinear interpolation layer and a separable 2D convolution layer. However, even a simple extension of these operations to 3D would require implementing a new custom operator in $\mathrm{C}++$ or CUDA to be linked with the main library. This can already be tedious and error-prone. Furthermore, while the forward algorithm is being developed, the adjoint must be re-derived by hand and kept in sync with the forward operator. This makes experimentation and prototyping especially difficult. Finally, both the forward and backward implementations ought to be reasonably optimized so that a model can be trained in a finite amount of time to verify its design.

We implemented a PyTorch backend for Halide so that our derivatives can be plugged into PyTorch's autograd system. We used this backend to re-implement custom operators recently proposed in the literature: the transformation layer in the spatial transformer network [Jaderberg et al. 2015], the warping layer in Flownet 2.0 [Ilg et al. 2017], and the bilateral slicing layer in deep bilateral learning [Gharbi et al. 2017]. The performance of our automatically scheduled code matches highly-optimized primitives written in CUDA, and is much faster than unoptimized code. We compare the runtime of our method to PyTorch, CNTK, and hand-written CUDA code in Table 1.

5.1.1 Spatial transformer network. The spatial transformer network of Jaderberg et al.[2015] applies an affine warp to an intermediate feature map of a neural network.

The function containing the forward Halide code is 31 lines long excluding comments, empty lines, and function declarations. Due to the popularity of this operator, deep learning frameworks have implemented specialized functions for the layer. The cuDNN library [Chetlur et al. 2014] added its own implementation in version 5 (2016), a year after the original publication. It took another year for PyTorch to implement a wrapper around the cuDNN code. We compare our performance to PyTorch's grid_sample and affine_grid functions which use the cuDNN implementation on GPU. On $512 \times 512$ images with 16 channels and a batch size of 4 , our CPU code is around 2.3 times faster than PyTorch's implementation, and our GPU code is around 20 percent slower than the highly-optimized version implemented in cuDNN. Currently Halide does not support texture sampling on GPU, which could be causing some of the slowdown. We also compare our performance to a CNTK implementation of spatial transformer using the gather operation. Our GPU code is around 10 times faster than the CNTK implementation.
Table 1. Performance of our approach for custom neural network operators. The runtime measures end-to-end latency for forward+backward evaluation. The spatial transformer transforms a batch of $4 \times 16 \times 512 \times 512$. The Flownet node warps a batch of $4 \times 64 \times 512 \times 512$ images with a $2 \mathrm{D}$ warping field. The BilateralSlice layer processes images with size $4 \times 4 \times 1024 \times 1024$ and grid size $4 \times 12 \times 64 \times 64$. Measurements were made on an Intel Core i7-3770K CPU @ 3.50GHz, with 16GB of RAM and a NVIDIA Titan X (Pascal) GPU with 12 GB of RAM.

\begin{tabular}{lccc} 
operator & SpatialTransformer & Flownet & BilateralSlice \\
\hline PyTorch (cpu) & $1094 \mathrm{~ms}$ & $4240 \mathrm{~ms}$ & $19819 \mathrm{~ms}$ \\
ours $(\mathrm{cpu})$ & $461 \mathrm{~ms}$ & $2466 \mathrm{~ms}$ & $1957 \mathrm{~ms}$ \\
\hline PyTorch $(\mathrm{gpu})$ & $11 \mathrm{~ms}$ & $482 \mathrm{~ms}$ & $1440 \mathrm{~ms}$ \\
CNTK (gpu) & $136 \mathrm{~ms}$ & $404 \mathrm{~ms}$ & $270 \mathrm{~ms}$ \\
manual CUDA (gpu) & - & $181 \mathrm{~ms}$ & $430 \mathrm{~ms}$ \\
ours (gpu) & $13 \mathrm{~ms}$ & $178 \mathrm{~ms}$ & $64 \mathrm{~ms}$
\end{tabular}

Having fixed functions such as affine_grid can be problematic when users want to slightly modify their models and experiment with different ideas. For example, changing the interpolation scheme (e.g., bicubic or Lanczos instead of bilinear), or interpolating over more dimensions (e.g., transforming volume data) would require implementing a new custom operator. Using our system, these modifications only require minor code changes to the forward algorithm. Our system then generates the derivatives automatically, and our automatic scheduler provides performance without further effort.

5.1.2 Warping layer. FlowNet 2.0 [Ilg et al. 2017], which targets optical flow applications, introduced a new 2D warping layer. Compared to the previous spatial transformer layer, this warping layer is a more general transform using a per-pixel warp-field instead of a parametric transformation.

The function containing the forward Halide code is 18 lines long. The original warping function was implemented as a custom node in Caffe. The authors had to write the forward and reverse code for both the CPU and GPU backends. In total it comprises more than 400 lines of code $^{1}$. While the custom node can handle 2D warps well, adapting it to higher-dimensional warps or semi-parametric warps would be challenging. Our system makes this much easier. In addition to PyTorch and CNTK, we also compare the performance of our GPU code with a highly-optimized reimplementation from NVIDIA $^{2}$. The performance of our code is comparable to the highlyoptimized CUDA code.

5.1.3 Bilateral slicing layer. Deep bilateral learning [Gharbi et al. 2017] is a general, high-performance image processing architecture inspired by bilateral grid processing and local affine color transforms. It can be used to approximate complicated image processing pipelines with high throughput. The algorithm works by splatting a 2D image onto a 3D grid using a convolutional network. Each voxel of the grid contains an affine transformation matrix. A highresolution guidance map is then used to slice into the grid and produce a unique, interpolated, affine transform to apply to each

\footnotetext{
${ }^{1}$ FlowNet 2.0: https://github.com/lmb-freiburg/flownet2/blob/master/src/caffe/layers/ flow_warp_layer.cu

${ }^{2}$ Nvidia Flownet 2.0: https://github.com/NVIDIA/flownet2-pytorch
} 
input pixel. The original implementation in TensorFlow had to implement a custom node ${ }^{1}$ for the final slicing operation due to the lack of an efficient way to perform trilinear interpolation on the grid. This custom node also applies the affine transformation on the fly to avoid instantiating a high-resolution image containing all the affine parameters at each pixel. The reference custom node had around 300 lines of CUDA code excluding comments and empty lines. Using the recently introduced general scattering functionality, we can implement the same operation directly in PyTorch. Figure 2 shows a comparison between our Halide code, reference CUDA code, and PyTorch code.

The PyTorch and CNTK implementations are modestly more complex than our code. PyTorch is 20 times slower while CNTK is 4 times slower on an $1024 \times 1024$ input with a grid size of $32 \times$ $32 \times 8$ and a batch size of 4 . CNTK is faster than PyTorch due to different implementation choices on the gather operations. The manual CUDA code aims for clarity more than performance, but is both more complicated and 6.7 times slower than our code.

Gharbi et al. [2017] argue that training on high resolution images is key to capturing the high frequency features of the image processing algorithm being approximated. Both the PyTorch and CNTK code run out of memory on a $2048 \times 2048$ input with grid size $64 \times 64 \times 8$ on a Titan GPU with 12 GB of memory. This makes it almost impossible to experiment with high-resolution inputs. Our code is 13.7 times faster than the authors' reference implementation on this problem size.

\subsection{Parameter optimization for image processing pipelines}

Traditionally, when developing an image processing algorithm, a programmer manually tunes the parameters of their pipeline to make it work well on a small test set of images. When the number of parameters is large, manually determining these parameters becomes very difficult.

In contrast, modern deep learning methods achieve impressive results by using a large number of parameters and many training images. We demonstrate that it is possible to apply a similar strategy to general image processing algorithms, by augmenting the algorithm with more parameters, and tuning these parameters through an offline training process. Our system provides the necessary gradients for this optimization. Users write the forward code in Halide, and then optimize the parameters of the code using training images.

We demonstrate this with an image demosaicking algorithm based on the adaptive homogeneity-directed demosaicking of Hirakawa and Parks [2005], and a non-blind image deconvolution algorithm based on the sparse adaptive priors proposed by Fortunato and Oliveira [2014].

5.2.1 Image demosaicking. Demosaicking seeks to retrieve a fullcolor image from incomplete color samples captured through a color filter array, where each pixel only contains one out of three red, green and blue colors. Traditional demosaicking algorithms work well on most cases, but can exhibit structured aliasing artifacts such as zippering and moiré (Figure 5). Recent methods using deep learning have achieved impressive results [Gharbi et al. 2016],

$\overline{{ }^{1} \text { https://github.com/mgharbi/hdrnet/blob/master/hdrnet/ops/bilateral_slice.cu.cc }}$ (a) AHD

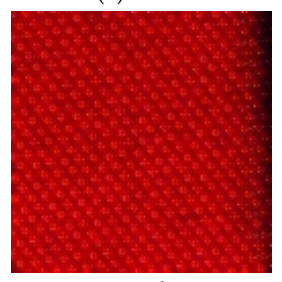

$19.6 \mathrm{~dB}$

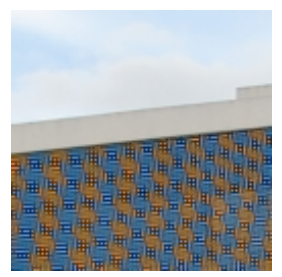

$19.7 \mathrm{~dB}$ (b) ours, $85 \times 5$ filters

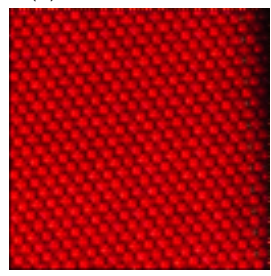

$24.7 \mathrm{~dB}$

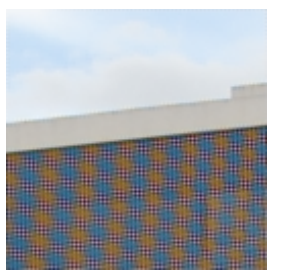

$21.4 \mathrm{~dB}$ (c) ground truth
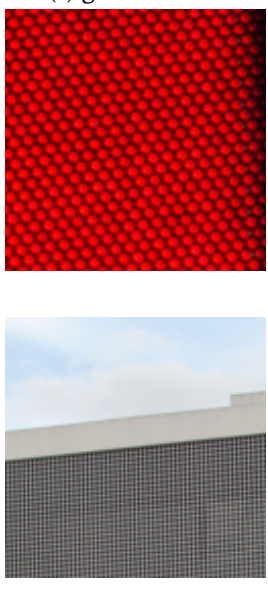

Fig. 5. We use our automatic gradients to relax the AHD demosaicking algorithm (a) by adding more filters to interpolate the green channel ( 8 instead of 2 here, with $5 \times 5$ footprint instead of $5 \times 1$ ). With this simple tweak, and by optimizing the filters using our automatically generated derivatives, we can obtain sharper images in difficult cases (b), first row. The smallfootprint of this simple demosaicking method nevertheless inherits some of the limitations of AHD. In particular, it leads to artifacts in complex, moiré-prone patterns (second row). Images taken from deep demosaicking dataset [Gharbi et al. 2016].

Table 2. PSNR for several demosaicking techniques following the evaluation methodology of Gharbi et al. (higher is better). We implemented a version of Hirakawa et al.'s AHD demosaicking algorithm with our system. Despite the simplicity of our approach, by relaxing the algorithm's specifications (i.e. adding more filters on the green channel reconstruction with larger footprints) and re-optimizing the parameters, we achieve higher fidelity (over 1 $\mathrm{dB}$ better) for a similar computational cost. While our method does not rival state-of-the-art deep-learning-based techniques, it is significantly faster and opens up new avenues to optimize more parsimoniously parametrized algorithms tailored to the problem. (Timings reported for a 1 megapixel image. $\left({ }^{*}\right)$ Timing for these algorithms is from non-optimized MATLAB code.)

\begin{tabular}{lccccr} 
& kodak & mcm & vdp & moiré & time \\
\hline bilinear & 32.9 & 32.5 & 25.2 & 27.6 & ${ }^{*} 127 \mathrm{~ms}$ \\
Adobe Camera Raw 9 & 33.9 & 32.2 & 27.8 & 29.8 & - \\
AHD Hirakawa [2005] & 36.1 & 33.8 & 28.6 & 30.8 & ${ }^{*} 1618 \mathrm{~ms}$ \\
ours (2 filters, 5x5) & 36.7 & 34.7 & 29.4 & 31.5 & $71 \mathrm{~ms}$ \\
ours (9 filters, 5x5) & 36.8 & 35.2 & 29.8 & 31.7 & $177 \mathrm{~ms}$ \\
ours (15 filters, 7x7) & 37.3 & 35.5 & 30.1 & 32.0 & $324 \mathrm{~ms}$ \\
Gharbi [2016] & 41.2 & 39.5 & 34.3 & 37.0 & $2932 \mathrm{~ms}$
\end{tabular}

however, the execution time is still an issue for practical usage. We relax the adaptive homogeneity-directed demosaicking algorithm (AHD) [Hirakawa and Parks 2005], variations of which are the default algorithms in Adobe Camera Raw and dcraw. We increase the number of filters to interpolate the green channel. We also fine-tune the chrominance (red-blue) interpolation filters from the AHD reference. We experiment with different number of filters and filter sizes to explore the runtime versus accuracy trade-off. We optimized 


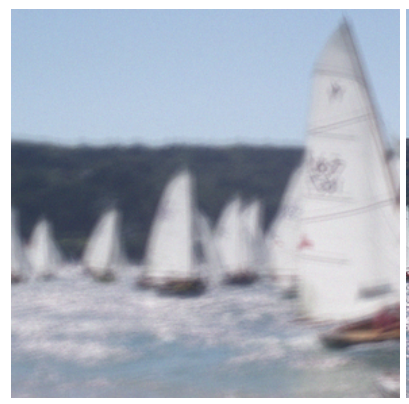

blurred

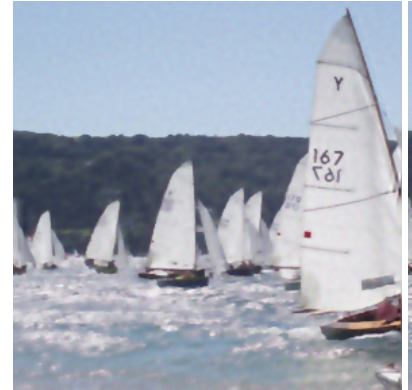

Fortunato $2014(25.39 \mathrm{~dB})$

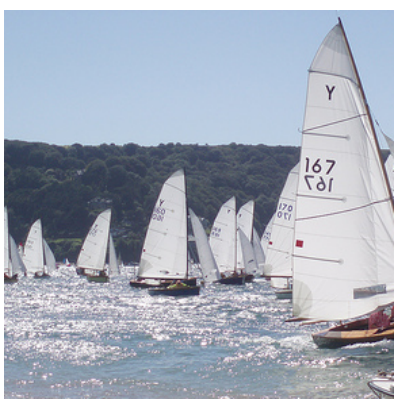

ground truth

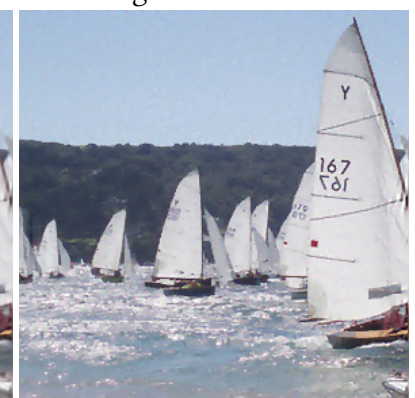

ours $(27.37 \mathrm{~dB})$
Fig. 6. We use automatic gradients to enhance Fortunato and Oliveira's non-blind deconvolution algorithm [2014]. We use more iterations and automatically train the weights, thresholds and filtering parameters. We are able to get sharper results. On eight randomly selected-images we achieve an average PSNR of $29.57 \mathrm{~dB}$. Using the original algorithm with its original parameters the PSNR is $28.51 \mathrm{~dB}$. Image taken from ImageNet [Deng et al. 2009]

the filter weights on Gharbi et al.'s [2016] training dataset using the gradients provided by our system. The results are illustrated in Table 2. With this simple modification, we obtain a significant 1 to $1.5 \mathrm{~dB}$ improvement on the more difficult datasets (moiré and $v d p$ ), depending on the number of filters used. We also obtain visually sharper images on many challenging cases, as shown in Figure 5.

With its limited footprint and filtering complexity, our optimized demosaicking still struggles on moiré-prone textures. Our system will allow users to experiment with more complex ideas without having to implement the derivatives at each step. For instance, we were able to quickly experiment with (and ultimately discard) alternative algorithms (e.g. using filters that take the ratio between colors into account and 1D directional filters).

5.2.2 Non-blind image deconvolution. The task of non-blind image deconvolution is: given a point spread function and a blurry image, which is the result of a latent natural image convolved with the function, recover the underlying image. The problem is highly ill-posed, therefore the quality of the reconstruction heavily depends on the priors we place on the image. It is thus important to learn a good set of parameters for those priors.

We based our implementation on the sparse adaptive prior proposed by Fortunato and Oliveira [2014]. The original method works in a 2-stage fashion. In the first stage they solve a conventional $L^{2}$
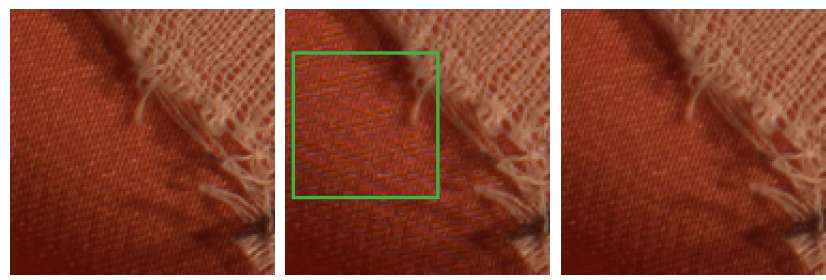

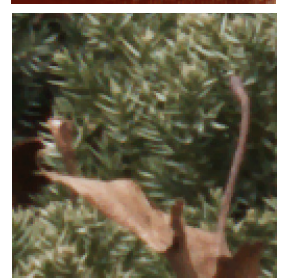

(a) our output $R$

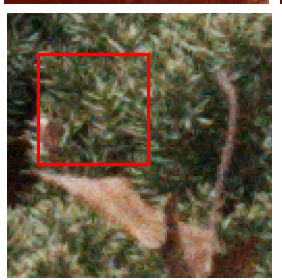

(b) dcraw (AHD) single frame

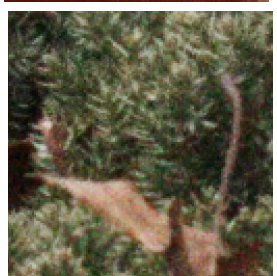

(c) [Gharbi 2016] single frame
Fig. 7. Automatic gradients can be used for inverse problems such as highresolution demosaicking from a burst of images. The user only needs to implement the forward model. Bursts of RAW images are captured with a Nikon D810 camera then jointly aligned and demosaicked (13 and 23 images respectively, only showing crops). We initialize our recontruction to a simple bilinear interpolation (not shown) and solve an inverse problem to recover both a set of homographies and a demosaicked image that matches the captured data when reprojected. Compared to the result of dcraw's AHD algorithm (a) and Gharbi et al. [2016] (c), our output (b) is much sharper, and shows less noise (red square) and color moiré (green square).

deconvolution using a set of discrete derivative filters as the prior. Then they use an edge-aware filter to cleanup the noise in the image. In the second stage, another $L^{2}$ deconvolution is solved for large discrete derivatives by matching the prior terms to the result of the first stage, masked by a smooth thresholding function.

We extend the method by increasing the number of stages (we use 4 instead of 2), and having a different set of filters for the priors for each stage. We optimize the weights of the prior filters, the smoothness parameters of the edge-aware filter (we use a bilateral grid), and the thresholding parameters in the smooth thresholding functions.

To demonstrate the ability of our system to handle nested derivatives, we implemented a generic non-linear conjugate gradient solver using a linear search algorithm based on Newton-Raphson to solve for the $L^{2}$ deconvolution. We write the conjugate gradient loop in PyTorch, but implement the gradient and vector-Hessian-vector product (required in the line search step) in Halide. We also implemented the bilateral grid filtering step in Halide. To optimize the parameters, we then differentiate through the gradients we used for the non-linear conjugate gradient algorithm. We train our method on ImageNet [Deng et al. 2009] and use the point spread function generation scheme described in Kupyn et al.'s work [2017]. We initialize the parameters to the recommended parameters described in Fortunato and Oliveira's work. Figure 6 shows the result. 


\subsection{Inverse imaging problems: optimizing for the image}

The derivatives produced by our automatic differentiation algorithm can be readily employed to solve inverse problems in computational photography. Using our system, users can quickly experiment with different forward models or different priors. We demonstrate this on a burst-demosaicking inverse pipeline.

Given $N$ misaligned Bayer RAW images, our goal is to reconstruct a full color image as well as estimate the homography parameters that align our reconstruction to the input data. We do this by minimizing the following cost function:

$$
\min _{R, H_{i}} \sum_{i=1}^{N}\left\|M H_{i} R-I_{i}\right\|_{2}^{2}+\lambda\|\nabla R\|_{1}
$$

where $M$ decimates the color samples according to the Bayer mosaic pattern. The homographies $H_{i}$ align our reconstruction $R$ to the input data $I_{i}$.

Gradient descent can help us minimize the function locally, but Equation 1 is highly non-convex, so a good initialization is critical. We initialize the $H_{i}$ using RANSAC [Fischler and Bolles 1981] and SIFT-based features [Lowe 2004] in a pairwise fashion. We also initialize $R=I_{0}$. This part is implemented in OpenCV ${ }^{1}$. From this starting point, we jointly refine the alignment and our estimate of the full-color image by minimizing the loss function (1). Compared to any individual image $I_{i}$, our reconstruction is sharper, and does not suffer from color moiré artifacts (Figure 7). We use the ADAM gradient-descent optimizer [Kingma and $\mathrm{Ba} 2014$ ] for 300 iterations, setting the learning rate to $10^{-2}$ for $R$ and $10^{-4}$ for $H_{i}$. Our algorithm provides the gradient of the loss with respect to the reconstructed image $R$ and homographies $H_{i}$. We set $\lambda=10^{-3}$. For $132048 \times 2048$ images, computing the initial homographies takes $44.5 \mathrm{~s}$, initializing the reconstruction $0.1 \mathrm{~s}$. Minimizing the cost function takes $179.4 \mathrm{~s}$ using the code generated by our automatic scheduler on a Titan X (Pascal) GPU.

\subsection{Lens optimization}

While we focus on imaging, Halide can express any feed-forward pipeline of arithmetic on multi-dimensional arrays. There are numerous non-imaging applications in this class, and taking derivatives is useful for many of them. As an example, we implemented a simple ray-tracer for a system of spherical lenses in Halide, and used our system to construct derivatives of the sharpness with respect to the lens positions and curvatures. In Figure 8, we start from an existing Zeiss design [Lange 1957] and reoptimize it to be more compact while maintaining the field of view, F-number, and sharpness.

\subsection{Future work}

As these applications demonstrate, our system automatically delivers state of the art performance when computing the gradients of general image processing pipelines. We see two major directions for future work.

Higher-order derivatives and non-scalar outputs. Some optimization methods require derivatives of non-scalar outputs, the full Hessian matrix, or even higher-order derivatives [Girolami and

\footnotetext{
$\overline{{ }^{1} \text { OpenCV: https://github.com/opencv/opencv }}$
}

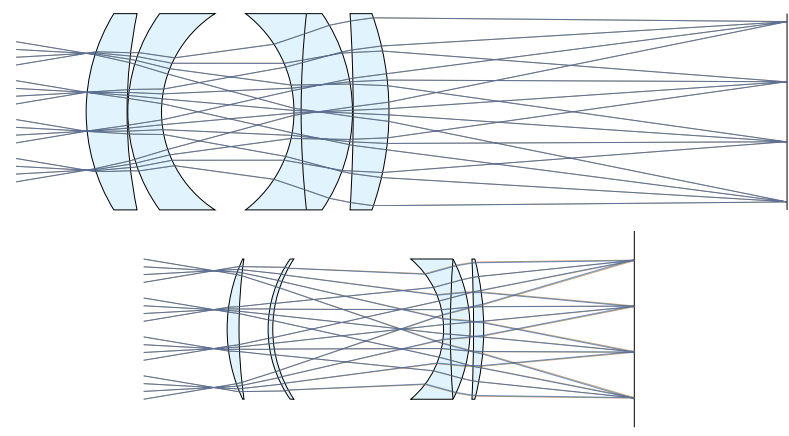

Fig. 8. Halide augmented with gradients is useful for a wider range of applications than just image processing and machine learning. By expressing a ray-tracer for an optical system in Halide and taking derivatives of sharpness with respect to the lens parameters, we can reoptimize a classic Zeiss lens design [Lange 1957] (above) to be more compact (below) while maintaining as much sharpness as possible.

Calderhead 2011]. Our system supports repeated or nested application of differentiation. However, it only differentiates with respect to one scalar at a time. When the dimensionality of both the input and the output are high, there are automatic differentiation algorithms that are more efficient than both forward- and reversemode (e.g., vertex elimination [Griewank and Reese 1991] or the $\mathrm{D}^{*}$ algorithm [Guenter 2007]). Incorporating these algorithms into our system, and developing better interfaces for non-scalar outputs and higher-order derivatives, will broaden the range of possible applications.

Better automatic scheduling. While it is possible to manually schedule the synthesized reverse computation, we found it challenging for non-trivial examples, and relied on our automatic scheduler entirely for this work. Its performance is good for gradient pipelines, but inspecting the generated code reveals plenty of room for further improvement. We consider the general Halide automatic scheduling problem still unsolved.

\section{CONCLUSION}

Gradient-based optimization is revolutionizing many fields including image processing, but the efficient computation of derivatives has so far been difficult, requiring one to either conform to limited building blocks or to error-prone manual derivation and challenging performance optimization. In contrast, our method can automatically generate high-performance gradient code for general image processing pipelines. Our method only requires the implementation of forward operators in a language that is concise, easy to maintain, and portable. It then automatically derives the gradient code using reverse automatic differentiation. We have presented a new automatic performance tuner that handles the particular computation patterns exhibited by derivatives. Our code compiles to a variety of platforms such as x86, ARM and GPUs, which is critical both for final deployment and for efficient training.

We have demonstrated that our work enables several types of applications, from custom neural network nodes, to the tuning of 
internal image processing parameters, to the solution of inverse problems. It dramatically simplifies the exploration of custom neural network nodes by automatically providing a level of performance that has so far been reserved to advanced CUDA programmers. It makes it easy to optimize internal weights and parameters for general image-processing pipelines, a step that few practitioners feel they can afford due to the cost of implementing gradients, which is especially true during the algorithmic exploration stages. Our system can also be used for inverse problems (which can even include unknown imaging parameters in addition to the unknown image) The user now only needs to worry about implementing the forward model. Each of the demonstrated applications was implemented initially in a few hours, and then evolved rapidly, with correct gradients and high-performance implementation automatically provided at each step by our method. We believe this will create new opportunities for rapid research and development in learning- and optimization-based imaging applications.

\section{ACKNOWLEDGMENTS}

This work is partially funded by Toyota, the Intel Science and Technology Center for Agile Design, ADEPT Lab industrial sponsors Google, Siemens, and SK Hynix, and the NSF/Intel Partnership on Computer Assisted Programming for Heterogeneous Architectures through grant CCF-1723445.

\section{REFERENCES}

Martín Abadi, Ashish Agarwal, Paul Barham, Eugene Brevdo, Zhifeng Chen, Craig Citro, Greg S. Corrado, Andy Davis, Jeffrey Dean, Matthieu Devin, Sanjay Ghemawat, Ian Goodfellow, Andrew Harp, Geoffrey Irving, Michael Isard, Yangqing Jia, Rafal Jozefowicz, Lukasz Kaiser, Manjunath Kudlur, Josh Levenberg, Dan Mané, Rajat Monga, Sherry Moore, Derek Murray, Chris Olah, Mike Schuster, Jonathon Shlens, Benoit Steiner, Ilya Sutskever, Kunal Talwar, Paul Tucker, Vincent Vanhoucke, Vijay Vasudevan, Fernanda Viégas, Oriol Vinyals, Pete Warden, Martin Wattenberg, Martin Wicke, Yuan Yu, and Xiaoqiang Zheng. 2015. TensorFlow: Large-Scale Machine Learning on Heterogeneous Systems.

Jonathan T Barron and Ben Poole. 2016. The fast bilateral solver. In European Conference on Computer Vision. Springer, 617-632.

James Bergstra, Olivier Breuleux, Frédéric Bastien, Pascal Lamblin, Razvan Pascanu, Guillaume Desjardins, Joseph Turian, David Warde-Farley, and Yoshua Bengio. 2010. Theano: a CPU and GPU Math Expression Compiler. In Proceedings of the Python for Scientific Computing Conference (SciPy).

Christian Bischof, Alan Carle, George Corliss, and Andreas Griewank. 1992. ADIFOR Automatic Differentiation in a Source Translator Environment. In Papers from the International Symposium on Symbolic and Algebraic Computation (ISSAC '92). 294302.

Vladimir Bychkovsky, Sylvain Paris, Eric Chan, and Frédo Durand. 2011. Learning Photographic Global Tonal Adjustment with a Database of Input / Output Image Pairs. In The Twenty-Fourth IEEE Conference on Computer Vision and Pattern Recognition.

Jiawen Chen, Sylvain Paris, and Frédo Durand. 2007. Real-time Edge-aware Image Processing with the Bilateral Grid. ACM Trans. Graph. (Proceedings of SIGGRAPH) 26, 3, Article 103 (July 2007).

Sharan Chetlur, Cliff Woolley, Philippe Vandermersch, Jonathan Cohen, John Tran, Bryan Catanzaro, and Evan Shelhamer. 2014. cuDNN: Efficient Primitives for Deep Learning. arXiv preprint arXiv:1410.0759 (2014).

J. Deng, W. Dong, R. Socher, L.-J. Li, K. Li, and L. Fei-Fei. 2009. ImageNet: A Large-Scale Hierarchical Image Database. In CVPR09.

Zachary Devito, Michael Mara, Michael Zollhöfer, Gilbert Bernstein, Jonathan RaganKelley, Christian Theobalt, Pat Hanrahan, Matthew Fisher, and Matthias Niessner 2017. Opt: A Domain Specific Language for Non-Linear Least Squares Optimization in Graphics and Imaging. ACM Trans. Graph. 36, 5, Article 171 (Oct. 2017), 27 pages.

Martin A. Fischler and Robert C. Bolles. 1981. Random Sample Consensus: A Paradigm for Model Fitting with Applications to Image Analysis and Automated Cartography. Commun. ACM 24, 6 (June 1981), 381-395.

Horacio E. Fortunato and Manuel M. Oliveira. 2014. Fast high-quality non-blind deconvolution using sparse adaptive priors. The Visual Computer 30, 6-8 (2014), 661-671.
Leon A Gatys, Alexander S Ecker, and Matthias Bethge. 2016. Image style transfer using convolutional neural networks. In Proceedings of the IEEE Conference on Computer Vision and Pattern Recognition. 2414-2423.

Michaël Gharbi, Gaurav Chaurasia, Sylvain Paris, and Frédo Durand. 2016. Deep Joint Demosaicking and Denoising. ACM Trans. Graph. (Proceedings of SIGGRAPH Asia) 35, 6, Article 191 (Nov. 2016), 12 pages.

Michaël Gharbi, Jiawen Chen, Jonathan T Barron, Samuel W Hasinoff, and Frédo Durand. 2017. Deep bilateral learning for real-time image enhancement. ACM Trans. Graph. (Proceedings of SIGGRAPH) 36, 4 (2017), 118.

Mark Girolami and Ben Calderhead. 2011. Riemann manifold langevin and hamiltonian monte carlo methods. Fournal of the Royal Statistical Society: Series B (Statistical Methodology) 73, 2 (2011), 123-214.

Andreas Griewank, David Juedes, and Jean Utke. 1996. Algorithm 755: ADOL-C: A Package for the Automatic Differentiation of Algorithms Written in $\mathrm{C} / \mathrm{C}++$. ACM Trans. Math. Softw. 22, 2 (June 1996), 131-167.

Andreas Griewank and Shawn Reese. 1991. On the Calculation of Jacobian Matrices by the Markowitz Rule. In Automatic Differentiation of Algorithms: Theory, Implementation, and Application, Andreas Griewank and George F. Corliss (Eds.). $126-135$.

Andreas Griewank and Andrea Walther. 2008. Evaluating Derivatives: Principles and Techniques of Algorithmic Differentiation (second ed.). Society for Industrial and Applied Mathematics.

Brian Guenter. 2007. Efficient Symbolic Differentiation for Graphics Applications. ACM Trans. Graph. (Proceedings of SIGGRAPH) 26, 3 (July 2007).

Laurent Hascoet and Valérie Pascual. 2013. The Tapenade Automatic Differentiation Tool: Principles, Model, and Specification. ACM Trans. Math. Softw. 39, 3, Article 20 (May 2013), 43 pages.

Felix Heide, Steven Diamond, Matthias Niessner, Jonathan Ragan-Kelley, Wolfgang Heidrich, and Gordon Wetzstein. 2016. ProxImaL: Efficient Image Optimization Using Proximal Algorithms. ACM Trans. Graph. (Proceedings of SIGGRAPH) 35, 4, Article 84 (July 2016), 15 pages.

Felix Heide, Markus Steinberger, Yun-Ta Tsai, Mushfiqur Rouf, Dawid Pająk, Dikpal Reddy, Orazio Gallo, Jing Liu, Wolfgang Heidrich, Karen Egiazarian, Jan Kautz, and Kari Pulli. 2014. FlexISP: A Flexible Camera Image Processing Framework. ACM Trans. Graph. (Proceedings of SIGGRAPH) 33, 6, Article 231 (Nov. 2014), 13 pages.

Keigo Hirakawa and Thomas W. Parks. 2005. Adaptive homogeneity-directed demosaicing algorithm. IEEE Trans. Image Processing 14, 3 (2005), 360-369.

Robin J. Hogan. 2014. Fast Reverse-Mode Automatic Differentiation Using Expression Templates in C++. ACM Trans. Math. Softw. 40, 4, Article 26 (July 2014), 16 pages.

Berthold KP Horn and Brian G Schunck. 1981. Determining optical flow. Artificial intelligence 17, 1-3 (1981), 185-203.

Satoshi Iizuka, Edgar Simo-Serra, and Hiroshi Ishikawa. 2016. Let there be color!: joint end-to-end learning of global and local image priors for automatic image colorization with simultaneous classification. ACM Transactions on Graphics (TOG) 35, 4 (2016), 110.

E. Ilg, N. Mayer, T. Saikia, M. Keuper, A. Dosovitskiy, and T. Brox. 2017. FlowNet 2.0: Evolution of Optical Flow Estimation with Deep Networks. In IEEE Conference on Computer Vision and Pattern Recognition (CVPR).

Max Jaderberg, Karen Simonyan, Andrew Zisserman, et al. 2015. Spatial transformer networks. In Advances in Neural Information Processing Systems. 2017-2025.

Yangqing Jia, Evan Shelhamer, Jeff Donahue, Sergey Karayev, Jonathan Long, Ross Girshick, Sergio Guadarrama, and Trevor Darrell. 2014. Caffe: Convolutional Architecture for Fast Feature Embedding. In Proceedings of the 22Nd ACM International Conference on Multimedia (MM '14). 675-678.

Diederik Kingma and Jimmy Ba. 2014. Adam: A method for stochastic optimization. arXiv preprint arXiv:1412.6980 (2014).

Orest Kupyn, Volodymyr Budzan, Mykola Mykhailych, Dmytro Mishkin, and Jiri Matas 2017. DeblurGAN: Blind Motion Deblurring Using Conditional Adversarial Networks. arXiv preprint arXiv:1711.07064 (2017).

Leslie Lamport. 1975. The Hyperplane Method for an Array Computer. In Proceedings of the Sagamore Computer Conference on Parallel Processing. 113-131.

Gunther Lange. 1957. Gauss type photographic objective containing two outer collective and two inner dispersive members. U.S. Patent 2,799,207 A.

Seppo Linnainmaa. 1970. The representation of the cumulative rounding error of an algorithm as a Taylor expansion of the local rounding errors. Master's thesis. Univ. Helsinki.

David G. Lowe. 2004. Distinctive Image Features from Scale-Invariant Keypoints. Int. 7 . Comput. Vision 60, 2 (Nov. 2004), 91-110.

Fujun Luan, Sylvain Paris, Eli Shechtman, and Kavita Bala. 2017. Deep Photo Style Transfer. arXiv preprint arXiv:1703.07511 (2017).

Ravi Teja Mullapudi, Andrew Adams, Dillon Sharlet, Jonathan Ragan-Kelley, and Kayvon Fatahalian. 2016. Automatically Scheduling Halide Image Processing Pipelines. ACM Trans. Graph. (Proceedings of SIGGRAPH) 35, 4, Article 83 (July 2016), 11 pages.

Ravi Teja Mullapudi, Vinay Vasista, and Uday Bondhugula. 2015. PolyMage: Automatic Optimization for Image Processing Pipelines. SIGARCH Comput. Archit. News 43, 1 
(March 2015), 429-443.

Adam Paszke, Sam Gross, Soumith Chintala, Gregory Chanan, Edward Yang, Zachary DeVito, Zeming Lin, Alban Desmaison, Luca Antiga, and Adam Lerer. 2017. Automatic differentiation in PyTorch. (2017).

Jonathan Ragan-Kelley, Andrew Adams, Sylvain Paris, Marc Levoy, Saman Amarasinghe, and Frédo Durand. 2012. Decoupling Algorithms from Schedules for Easy Optimization of Image Processing Pipelines. ACM Trans. Graph. (Proceedings of SIGGRAPH) 31, 4, Article 32 (July 2012), 12 pages.

Jonathan Ragan-Kelley, Connelly Barnes, Andrew Adams, Sylvain Paris, Frédo Durand, and Saman Amarasinghe. 2013. Halide: A Language and Compiler for Optimizing Parallelism, Locality, and Recomputation in Image Processing Pipelines. SIGPLAN Not. 48, 6 (June 2013), 519-530.

S. Roth and M. J. Black. 2005. Fields of Experts: A framework for learning image priors In IEEE Conf. on Computer Vision and Pattern Recognition, Vol. 2. 860-867.

Leonid I Rudin, Stanley Osher, and Emad Fatemi. 1992. Nonlinear total variation based noise removal algorithms. Physica D: Nonlinear Phenomena 60, 1-4 (1992), 259-268.

D. E. Rumelhart, G. E. Hinton, and R. J. Williams. 1986. Parallel Distributed Processing: Explorations in the Microstructure of Cognition, Vol. 1. Chapter Learning Internal Representations by Error Propagation, 318-362.

Patricia Suriana, Andrew Adams, and Shoaib Kamil. 2017. Parallel Associative Reductions in Halide. In Proceedings of the 2017 International Symposium on Code Generation and Optimization (CGO '17).

Dmitry Ulyanov, Andrea Vedaldi, and Victor Lempitsky. 2017. Deep Image Prior. arXiv preprint arXiv:1711.10925 (2017)

Yu. M. Volin and G. M. Ostrovskii. 1985. Automatic computation of derivatives with the use of the multilevel differentiating technique - I: Algorithmic basis. Computers and Mathematics with Applications 11 (1985), 1099-1114.

Paul J Werbos. 1982. Applications of advances in nonlinear sensitivity analysis. In System modeling and optimization. Springer, 762-770.

Alexander B Wiltschko, Bart van MerriÃńnboer, and Dan Moldovan. 2017. Tangent: automatic differentiation using source code transformation in Python.

Li Xu, Jimmy Ren, Qiong Yan, Renjie Liao, and Jiaya Jia. 2015. Deep edge-aware filters. In Proceedings of the 32nd International Conference on Machine Learning (ICML-15) 1669-1678.

Yuting Yang, Sam Prestwood, and Connelly Barnes. 2016. VizGen: Accelerating Visual Computing Prototypes in Dynamic Languages. ACM Trans. Graph. (Proceedings of SIGGRAPH Asia) 35, 6, Article 206 (Nov. 2016), 13 pages.

Dong Yu, Adam Eversole, Mike Seltzer, Kaisheng Yao, Oleksii Kuchaiev, Yu Zhang, Frank Seide, Zhiheng Huang, Brian Guenter, Huaming Wang, Jasha Droppo, Geoffrey Zweig, Chris Rossbach, Jie Gao, Andreas Stolcke, Jon Currey, Malcolm Slaney, Guoguo Chen, Amit Agarwal, Chris Basoglu, Marko Padmilac, Alexey Kamenev, Vladimir Ivanov, Scott Cypher, Hari Parthasarathi, Bhaskar Mitra, Baolin Peng, and Xuedong Huang. 2014. An Introduction to Computational Networks and the Computational Network Toolkit. Technical Report.

Kai Zhang, Wangmeng Zuo, Yunjin Chen, Deyu Meng, and Lei Zhang. 2017. Beyond a gaussian denoiser: Residual learning of deep cnn for image denoising. IEEE Transactions on Image Processing (2017).

Richard Zhang, Phillip Isola, and Alexei A Efros. 2016. Colorful image colorization. In European Conference on Computer Vision. Springer, 649-666.

Barbara Zitova and Jan Flusser. 2003. Image registration methods: a survey. Image and vision computing 21, 11 (2003), 977-1000. 